\title{
Views of general practitioners on providing contraceptive advice and long-acting reversible contraception at the 6-week postnatal visit: a qualitative study
}

\author{
Hannah Lunniss, ${ }^{1}$ Sharon Cameron, ${ }^{2,3}$ Zhong E Chen ${ }^{4}$
}

\begin{abstract}
- Additional material is published online only. To view please visit the journal online (http://dx.doi.org/10.1136/jpprhc2015-101198)

${ }^{1}$ Medical Student, College of Medicine and Veterinary Medicine, University of Edinburgh, Edinburgh, UK ${ }^{2}$ Consultant Gynaecologist, Chalmers Sexual and Reproductive Health Service, NHS Lothian, Edinburgh, UK ${ }^{3}$ Royal Infirmary of Edinburgh, Edinburgh, UK

${ }^{4}$ Clinical Researcher, Chalmers Sexual and Reproductive Health Service, NHS Lothian, Edinburgh, UK
\end{abstract}

\section{Correspondence to}

Ms Hannah Lunniss, College of Medicine and Veterinary Medicine, University of Edinburgh, 47 Little France Crescent, Edinburgh EH16 4TJ, UK; s1111173@sms.ed.ac.uk

Received 20 February 2015 Revised 27 June 2015 Accepted 28 July 2015 Published Online First 26 August 2015

CrossMark

To cite: Lunniss $\mathrm{H}$, Cameron S, Chen ZE. J Fam Plann Reprod Health Care 2016;42:99-106.

\begin{abstract}
Background Increased uptake of long-acting reversible contraception (LARC) postpartum could prevent more unintended pregnancies and short inter-birth intervals. General practitioners (GPs) play a pivotal role in providing postpartum contraception at the 6-week postnatal visit. Aim To explore how GPs view their role in delivering postpartum contraception at the 6-week visit and on providing LARC at this time. Methods In-depth, semi-structured interviews with a purposive sample of 13 GPs in Edinburgh and the surrounding region in Scotland. The interviews were audio recorded, transcribed and thematically analysed.
\end{abstract}

Results All GPs confirmed that contraception was routinely discussed at the postpartum visit, although this was usually the last item covered. Most felt that while 6-weeks postpartum was adequate for most women to commence contraception, it was often too late for young mothers (aged under 20 years) or women from deprived areas. GPs provided prescriptions for oral contraception at this visit, but insertion of a contraceptive implant required a further appointment. For intrauterine contraception, women typically required two additional visits to the GP (for counselling and then insertion) or were referred to a local sexual health service. Some GPs saw their role as the main provider of postpartum contraception, whereas others felt they complemented the actions of midwives and health visitors.

Conclusions This study demonstrated that although contraception is discussed at a routine 6-week postpartum visit with a GP, there are delays for women wishing to commence LARC that create scope for unintended pregnancy. Strategies to facilitate access to LARC postpartum should be explored.
Key message points

- Increased uptake of long-acting reversible contraception (LARC) postpartum could prevent more unintended pregnancies that end in termination or short inter-birth intervals.

- A discussion about contraception is usually the last item covered at the 6-week postpartum visit to the general practitioner (GP).

- The need for detailed counselling, further appointments and long waiting times in some GP practices are perceived as barriers to women accessing LARC in the postpartum period.

\section{INTRODUCTION}

In 2013, just under 800000 women gave birth in the UK. ${ }^{1}$ Studies show that $50 \%$ of postpartum women have resumed intercourse by 6 weeks. ${ }^{2}$ Ovulation can occur in women who are not fully breastfeeding by 4 weeks and Scottish statistics show that only approximately one in four women are exclusively breastfeeding by 6 weeks. ${ }^{34}$ Clearly these statistics suggest that a sizeable proportion of the UK's postpartum population require effective contraception in the early postpartum period.

In the UK, women are offered a routine 6-week postpartum visit with their general practitioner (GP) which covers a range of issues relating to the mother's health in the postpartum period. During this visit, GPs play an 
important role in meeting women's contraceptive needs both in terms of offering advice and options as well as providing prescriptions and fitting contraceptive devices. Although there are opportunities prior to discharge from the maternity services for midwives to speak with mothers about the use of ongoing contraception, these conversations may not always take place. A recent Scottish study suggests that some midwives lack the confidence, doubt their competence and question the usefulness of providing contraceptive advice at this time. ${ }^{5}$ Moreover, most viewed their role as signposting women to their own GPs to discuss their contraceptive needs during the 6-week postnatal visit. Consistent with this, an online survey of UK mothers reported that for over half of the respondents, the 6-week GP visit was the first time contraception was properly discussed postpartum. ${ }^{6}$

Unintended pregnancy is a major public health issue. In 2013, 185331 pregnancies in England and Wales ended in termination of pregnancy. ${ }^{7}$ The recent UK National Survey of Sexual Attitudes and Lifestyles (NATSAL 3) published in 2013 reported that almost half the pregnancies in women aged under 20 years were unintended. ${ }^{8}$ Furthermore, it is estimated that just under one-third of all pregnancies proceeding to birth in the UK are originally unintended. ${ }^{9}$ Unintended pregnancies that continue to term are associated with higher incidences of depression and other mental illness in the mother, as well as poor mother-child relationships. ${ }^{10}$ Furthermore, short inter-pregnancy intervals are risk factors for neonatal death and preterm delivery. ${ }^{11}$ This highlights the importance of providing high-quality contraceptive advice and provision of the most effective methods to mothers postpartum.

Little research has been done on the views and experiences of GPs in providing postpartum contraception despite the key role they play in meeting a woman's reproductive health needs. There is also only limited recent literature on postpartum contraception in the UK, perhaps reflecting that this has been a neglected area of health care.

The aim of this study was to explore the views and experiences of GPs providing postpartum contraception, particularly long-acting reversible contraception (LARC). We especially wanted to explore GPs' views on the timing of the postpartum visit and its impact on preventing unplanned pregnancies, and their role in providing postpartum contraception. We were also keen to discover whether discussions about contraception were raised, when this topic was raised, and how the GPs proceeded to provide women with their chosen method of contraception.

\section{METHODS}

\section{Sampling}

A range of methods were used to maximise the recruitment of GPs with experience of working with different patient characteristics. A list was compiled with details of all the GP practices in Edinburgh and the surrounding region (Lothian), and the associated deprivation category (DepCat) of the area in which these practices were situated (DepCat scores: a measure of deprivation/affluence by postcode). ${ }^{12}$ A subset of 20 practices was chosen which provided a geographical and socioeconomic distribution. Information about the study was emailed to the practice managers to distribute to the GPs in the practice. GPs interested in participating were invited to email the researcher (HL) to arrange an interview at a convenient time.

To augment this approach, GPs engaged in undergraduate teaching at the University of Edinburgh, working at the sexual health service in Edinburgh, and GPs attending a postgraduate training event were also invited to participate. A 'snowball' approach was used whereby participants were asked to recommend a GP colleague who could be contacted about participation.

\section{Data collection}

Semi-structured interview were conducted with GPs, which took place at their practice. Interviews lasted around half an hour and a topic guide (see online Supplementary Figure S1) was used to ensure key topics were discussed during the interview. The key topics for the topic guide were selected by the second author (SC) based on the research aims, a review of the existing literature, and her clinical experience in sexual and reproductive health care. GPs were encouraged to expand on the key topics in relation to their experience and views, and the topic guide was kept under review to ensure it reflected the issues being raised. Before each interview the participant was asked to read and sign a consent form.

The interviews were audio-recorded onto a digital Dictaphone and transcribed verbatim. At this point, all personal information was removed from the transcripts to ensure anonymity. The transcripts were kept on a password-protected computer.

Research and development permission was obtained from NHS Lothian. The ethics committee advised that approval was not required, as the study was considered to be an audit.

\section{Analysis}

The interview transcripts were thematically analysed by the first author (HL), following the six-phase process described by Braun and Clarke. ${ }^{13}$ An inductive approach to analysis was taken. Initial coding ${ }^{14}$ began after seven interviews had been completed. Codes were chosen in order to describe the different chunks of text in the transcripts, rather than being generated from the research questions and then applied to the text. ${ }^{13}$ The codes were then grouped into potential themes, which were reviewed in relation 
to the research questions, and finalised once all the data had been collected.

\section{RESULTS}

\section{Demographics}

Table 1 shows the characteristics of the 13 GPs interviewed. There were two male and 11 female GPs, of which two were locums. Interviewees ranged from having less than 1 year's experience as a GP to 29 years. The GPs came from 10 practices, of which five were situated in affluent areas (DepCat scores 1 and 2), four were in moderately deprived areas, and one was in an area of high deprivation (DepCat score 7$).{ }^{12}$

\section{Views on the 6-week visit}

Content of consultation

The frequency at which the GPs carried out a 6-week consultation ranged from two per week to one every few months. The topics that the GPs listed as something they would discuss with a woman during the consultation were similar across the sample. The account below by GP1 was typical, demonstrating the range of different areas covered in the short consultation:

\section{"I would cover, briefly, how mum is finding things since the birth. So, that would be feeding, sleeping, her mood particularly. And then physically, if she's still having any bleeding, pain, fever, anything like that, and then examination. I wouldn't examine everyone... Then looking at any complications, and investigating those further. And then the last thing I would come to is contraception. I would always make sure we talk about $i t$, but the time allowed for that would be variable depending on the other issues that we deal with." [GP1]}

Depending on the practice, either 10 or 20 minutes were allocated for this consultation. While this was considered sufficient in 'uncomplicated' cases, GPs often remarked that women would attend with multiple concerns that required addressing, leaving little time for the contraceptive discussion:

\section{"Either mums come in and they're absolutely fine and there's no issues, or they come in with a million issues and it can take longer." [GP7] \\ "It's only a 10-minute appointment, which can be quite tight." [GP6]}

Timing of the appointment

When asked whether they felt that 6 weeks postpartum was an appropriate time point to be addressing postpartum contraception, the GPs identified that they felt different groups of women had different needs.

All the GPs agreed that a 6-week time point was appropriate for addressing postpartum contraception for the majority of women, as it was felt that only a minority of mothers would have resumed having sex by 6 weeks.

"To be honest, I think most women say, 'That's not on my agenda at the minute, you know'... I've never had anybody who's said, 'Oh I've already started having sex again', to be honest with you." [GP6]

"I personally find, probably because most of the mums I see are slightly older, I find that most mums will burst out laughing when we start talking about contraception." [GP12]

GPs working in practices in the more deprived areas in our sample raised concerns that a significant proportion of mothers did not attend the 6-week visit or attended late despite follow up by the health visitor. In contrast, the GPs from affluent areas reported that almost all their patients attended the 6-week visit.

Table 1 Summary of characteristics of the general practitioners interviewed

\begin{tabular}{llllll}
\hline GP \# & $\begin{array}{l}\text { Experience as a } \\
\text { GP (years) }\end{array}$ & Gender & $\begin{array}{l}\text { Age } \\
\text { (years) }\end{array}$ & $\begin{array}{l}\text { Deprivation/affluence } \\
\text { of practice area }\end{array}$ & $\begin{array}{l}\text { Trained to } \\
\text { fit LARC }\end{array}$ \\
\hline 1 & 6 & Female & - & Affluent & N/A* \\
2 & 10 & Female & 41 & N/A* & Yes \\
3 & 1 & Female & 30 & Moderately deprived & No \\
4 & 16 & Female & 45 & Affluent & No \\
5 & 29 & Female & 58 & Moderately deprived & No \\
6 & 3 & Female & 32 & Affluent & No \\
7 & 14 & Female & 44 & Affluent & Affluent \\
8 & 25 & Male & 55 & Moderately deprived & Yes \\
9 & 1 & Female & 29 & Moderately deprived & Yes \\
10 & 10 & Female & 49 & Affluent & Very deprived \\
11 & 8 & Female & 36 & & Yes \\
12 & 15 & Male & 45 & 44 &
\end{tabular}

${ }^{*}$ GPs 2 and 3 are locums.

GP, general practitioner; LARC, long-acting reversible contraception. 
Also, six of the GPs felt that it was potentially too late for young mothers:

"Our trouble is not all women come for their 6-week check. We're in a deprived area and many forget all about coming." [GP13]

"I guess you could argue it's potentially a bit late for some women. I can certainly think of one or two quite young women who have got pregnant very quickly after having the first baby, and you wonder whether you might in some cases miss it." [GP11]

\section{Contraceptive consultation}

When asked, all the GPs said that contraception was always discussed during the 6-week visit, although one GP left it out of the list of topics covered initially. The GPs talked about different ways that the topic was introduced during the consultation, which included asking women about their future family plans and asking them open questions on their thoughts about contraception. Other GPs noted that it was the mothers who brought up the issue of contraception with them.

"I would usually ask if they're planning on having any more kids in the near future, because that would obviously dictate what sort of contraception we'd more focus on." [GP1]

"Sometimes people are asking about it and they already have an idea, some people just say, 'I wondered if I could get started back on the pill I was on before?" [GP11]

In terms of when during the consultation contraception was brought up, the majority (10) of the GPs said that usually it tended to be the last topic covered.

"I tend to talk about it last, but I think it's important. I mean, I wouldn't not always talk about it." [GP6]

"But I do at the very end... What I tend to word it is, we have to talk about contraceptive, it's not something you might think about now, but just in case this crops up have any ideas? That sort of thing, any thoughts?" [GP12]

Needs of different groups of women

The GPs had diverse views on the groups of women that they found most challenging to provide postpartum contraceptive advice to or that they viewed as having a greater need for contraception to be established quickly. The group referred to most often were young mothers, who were perceived to be more likely to become sexually active again quickly and thus at higher risk of unplanned pregnancy. Many GPs also spoke of 'chaotic' patients, who were less likely to follow instructions or present for follow up.

"I suppose more chaotic patients. Patients who perhaps you think - are they going to come back for an implant if you advise them about that? But if I give them the pill, are they going to take it?" [GP3]

Other groups of women mentioned by individual GPs as challenging to give contraceptive advice to postpartum included women who have English as their second language, women living in deprived areas, medically complex women, women with very large families already, wives of servicemen, drug users, and sex workers.

When asked about whether short inter-birth intervals were a problem, there was a clear division of GPs' views depending on the deprivation/affluence of their practice area. GPs working in more affluent areas did not feel that this was a problem in their patient population.

"Not really a problem actually, not that we have ... It's not something I've ever thought about so I guess it can't be a problem." [GP8]

Conversely, GPs working in deprived areas did consider short inter-birth intervals and unplanned pregnancies to be a significant problem. One felt that it was particularly challenging trying to address the needs of women who already had large families, but did not want contraception, and the potential for conflict between the GP's and the patient's agendas.

"I mean, many of our patients don't want it [contraception], they want another baby quite quickly ... They [short inter-birth intervals] happen, but the patient doesn't think of them as a problem necessarily." [GP13]

\section{Methods of contraception and LARC}

The interviews also covered the different methods of contraception that the GPs are prescribing to postpartum women; access to LARC; and how acceptable the GPs perceived LARC to be to their patients. All but one GP identified oral contraceptives as the method of postpartum contraception they believed they were providing to women most commonly. This was usually the progestogen-only pill.

"I'd say 50\% leave with a prescription; another 25\% or so might leave with a plan, as in come back for an implant, come back for a coil; maybe $25 \%$ or so will just say no, it's not an issue, or we're using condoms or whatever." [GP10]

One GP (GP13) whose practice was in a deprived area stated that she prescribed the contraceptive implant as frequently as the pill for postpartum women. Three GPs mentioned that they often prescribed oral contraceptives as an interim method for women considering or waiting for an intrauterine device (IUD) or implant - in some of the practices the wait could be quite considerable.

"And for some of them, who say 'We're thinking about an IUD or Nexplanon', I would then say 'Shall I give 
you a supply of pills to use in the meantime while you make up your mind?'” [GP5]

Provision of LARC: service set-up

Of the $10 \mathrm{GP}$ practices represented in our sample, seven were able to insert both contraceptive implants and IUDs, one could insert implants but not IUDs, and two fitted neither. GPs from practices that fitted implants were generally prepared to provide counselling about the implant during the 6-week check, so that a woman wanting an implant at this point would need to return for one further appointment to have the device inserted. Waiting times for the implant were all within 2 weeks. GP13's practice (in the most deprived area) was able to fit implants at the 6-week check.

Of the seven practices that provided intrauterine methods, six required women to make two further appointments (one for counselling, and another for fitting). GP10 (from a relatively deprived area) was able to do the counselling during the 6-week visit, so the woman would only need one further appointment. The waiting time for fitting an intrauterine method was generally longer, 2 or 3 months in some practices. This was attributed by some GPs to fewer clinicians being able to fit IUDs and that it takes longer during an appointment to fit an IUD compared to an implant. In one practice the wait for an IUD was 2 weeks. Other practices that did not fit either the IUD or the implant referred women to the local sexual and reproductive health service for this.

"I think the waiting time for the coils is about 2 months. Rods [implants], it's much quicker, because we have two points of delivery - the nurse and the doctor. And they're at the practice ready to go. Whereas with the coil there's a little bit of counselling and assessment, so that takes longer." [GP4]

All the GPs highlighted the need for multiple appointments as a barrier to some women accessing LARC. Also, all the GPs felt that older, more affluent mothers were better informed about the contraceptive options available to them and were better at accessing services.

\begin{abstract}
"For some of our patients you wouldn't imagine them going away and looking something up online, or reading things before they came in. Which clearly, more educated, more middle-class patients might be doing ... I think it does have an impact on skills in coming in for appointments at the right time, and making follow-up appointments and so on." [GP11]
\end{abstract}

Views on acceptability of LARC

When asked whether they thought LARC was acceptable to their patients, the majority of the GPs felt that women were becoming more open to using LARC than previously. Other GPs felt that patients were still wary of LARC. In addition, some of the GPs expressed their own negative views towards LARC.

"I think certainly, they seem to be becoming more popular in the practice ... So you sort of feel word is getting out and about, particularly among young women." [GP11]

"A lot of people are quite negative about some of the long-acting specifics, especially the implant I find, because of the irregular bleeding. So that might be more of a factor ... But certainly if they can't get it done that day, they might just go, 'Ah, look I'll just go for what I can get the quickest. "[GP3]

"If it were me, I'd think, uh, can't be bothered with all that. Far too much hassle." [GP7]

One view expressed by several GPs was that the discontinuation rate for implants was high if women were not given enough time to consider this contraceptive option, and so a delay in having a LARC method inserted postpartum could be an advantage:

"I feel I remove lots [of] implants where it was put in without the person really getting a chance to decide. They're saying, 'I didn't even want this, I didn't get a chance to decide'. So I think it's good to give them a chance to think about it." [GP13]

\section{Role in providing postpartum contraception and antenatal} contraceptive advice

Role of the GP and the multidisciplinary team

The GPs expressed a range of views on their role in delivering postpartum contraception. Seven believed that GPs should be the main health care professional with responsibility for postpartum contraception, because they have access to the mother's medical history and the ability to prescribe or arrange contraception.

"I think that it is definitely one of our roles. Often the mums will say 'The midwife gave me a leaflet, or the midwife... I spoke to the midwife about it'. But obviously we're doing the prescribing. So, no, I do think it's very much our role." [GP7]

The other six GPs felt that more of a multidisciplinary approach should be adopted, with health visitors in particular playing a larger role than at present. Many GPs felt that this was necessary due to the diminished involvement of GPs in both antenatal and postnatal care. All but one GP reported confidence in health visitors' level of knowledge to be able to give advice about postpartum contraception.

"I don't see why it has to be a GP to be honest. And the way things are going, it may be that we become less involved in postnatal care if things are normal and well ... I think it should be with the midwives, the health visitors, and anyone they see, and then obviously the GP at the 6-week check is almost the fall back, if they haven't got started with anything yet." [GP8] 
Role of the GP and the provision of antenatal contraceptive advice

The GPs' responses were mixed when asked whether they thought introducing contraceptive advice antenatally would improve uptake of postpartum contraception. Six GPs were generally positive about the idea. Some felt there would be no reason not to, but doubted it would have any impact. Others felt that women were too focused on the baby and the delivery, and that antenatal contraceptive advice would not be well received at this time.

\begin{abstract}
"Yeah, I think that it probably would be worth briefly mentioning it to people, perhaps giving them a leaflet then, than they do when they've got a 6-week-old baby." [GP1]

"So however much information you give her during pregnancy, she's going to be thinking about what'll the delivery going to be like, how am I going to manage a baby, what's going to happen to my life? So contraception's probably not going to be ... something they're going to be thinking about." [GP3]
\end{abstract}

Several GPs referred to the fact that antenatal care is now almost entirely delivered by midwives, so even if a policy to provide contraceptive advice antenatally was introduced, they did not think it would be the GP's role.

\section{DISCUSSION}

A key finding of this project is confirmation that contraception is discussed at the 6-week postnatal visit by all the GPs in our sample, in keeping with National Institute for Health and Care Excellence (NICE) guidelines. ${ }^{15}$ This is consistent with the expectations of midwives working in the same region, as previous research found that they generally deferred contraceptive counselling to the GP. ${ }^{5}$ Another key finding was the GPs' view on the timing of the postpartum check for the provision of contraception, with many believing that few women had resumed sexual activity by 6 weeks postpartum. This is in contrast to studies that suggest as many as $50 \%$ of women will have resumed sex by this time. ${ }^{2}$

None of the GPs gave an explanation as to why contraception tended to be covered last at the 6-week postpartum appointment. It seems doubtful that a GP would admit to not thinking that postpartum contraception was important in an interview on the subject, even with anonymity. It is interesting therefore that their actions (namely raising the topic of contraception last) do not necessarily reflect the importance that they profess to place on postpartum contraception. It is possible some GPs see contraception as something that they "have to talk about" (see full quote from GP6 earlier) at the 6-week check, as opposed to being integral to the visit.

Importantly, GPs identified younger women and women from deprived areas as being most at risk of having an unintended pregnancy shortly after giving birth, and having the greatest need for postpartum contraception. This is consistent with the findings of Glasier et al. ${ }^{16}$ in 1996, who reported that women who had become pregnant again with an unintended pregnancy within 1 year of delivery were younger, and less likely to be married/cohabiting compared to other women. Whilst the majority of GPs said they felt it was appropriate for them to be the main provider of postpartum contraception, clearly the recognition that women failing to attend constituted a group at high risk of unintended pregnancy would support a multidisciplinary approach to providing postpartum contraception, at least for this group of women. Indeed, this would be in keeping with national sexual heath frameworks and NICE guidance for contraceptive services for young people, which advise that young women in maternity units and those at risk of an unintended pregnancy should receive contraceptive supplies and provision of LARC before discharge from the maternity service. ${ }^{17-19}$

Another finding of our study was that GPs believe the most common method of postpartum contraception they provided was oral contraception. This is in keeping with national data showing that this is the most popular contraceptive method used in the UK, ${ }^{20}$ although it is significantly less effective than LARC methods. ${ }^{21}$ Neither breastfeeding, nor being 6 weeks postpartum, are contraindications to use of implants or IUDs, ${ }^{22}$ so it is not clear why GPs are not providing more LARC postpartum. Our study would suggest that GPs believe some women have negative views about LARC (as do some of the GPs). Our study also suggests that there are important process barriers in being able to provide an implant or IUD at the 6-week visit. The range of postpartum health issues to be covered in the limited consultation time does not permit detailed counselling about all available LARC methods.

Although GPs had mixed views on the potential impact of antenatal advice about contraception, counselling in the antenatal period could help overcome the issue of women not having sufficient time to consider LARC and may even avoid the extra 'counselling' visit in the postpartum period. Of course if we consider the clinical evidence then we find a multicountry (Scotland, South Africa, China) randomised controlled trial showing no difference in contraceptive uptake between women who received specialist antenatal counselling and those who did not. ${ }^{23}$ However, this study was undertaken in 1997 when LARC methods were not so enthusiastically promoted, and the current single-rod contraceptive implant and intrauterine systems were not yet in general use. Antenatal advice does, however, need to be coupled with easy access to supplies postpartum, particularly so when women have a young baby to care for, and are possibly struggling with breastfeeding and recovering from an operative delivery. ${ }^{24} \mathrm{~A}$ recent survey of 
postpartum mothers in a maternity service in Edinburgh found that although 13\% indicated they were actually considering using a LARC method, as many as $43 \%$ stated that they would have chosen to have a LARC method if it had been possible to have it inserted before being discharged from hospital after delivery. ${ }^{25}$

A retrospective cohort study examining all second births in Scotland between 1992 and 1998 reported that $5.4 \%$ of second pregnancies were conceived within 6 months of the first birth, and that this short inter-pregnancy interval was an independent risk factor for neonatal death and preterm delivery. ${ }^{11}$ There is evidence from observational studies in the USA and Australia that uptake of the contraceptive implant soon after childbirth is associated with fewer rapid repeat pregnancies among young women. ${ }^{26} 27$ This highlights the potential role that postpartum LARC has to play in preventing not only unintended pregnancy but also improving obstetric outcomes nationally.

\section{Study limitations}

A key strength of the study is the qualitative design that enabled a more in-depth exploration of the views of GPs on the provision of postpartum contraception at the 6-week check. Although small in scale, this is the first study to examine this issue from a GP's perspective.

The biggest limitation of this study is the small sample of GPs. Unfortunately the initial methodical approach to recruiting GPs described earlier did not in itself yield sufficient responses, hence the need to employ more opportunistic recruitment methods. Also, female GPs, and GPs with a special interest in contraception, were more likely to respond and agree to be interviewed than other GPs. Therefore, the sampled GPs are likely to be better informed about postpartum contraception than the average GP. Another weakness is that this study only sampled GPs from one region in Scotland, and so may not necessarily reflect primary care practice across the whole of the UK. However, despite these issues, the study sample comprises a diverse sample of GPs and includes the views of GPs working in areas of varying levels of deprivation, providing different perspectives on contraceptive needs among their postpartum populations.

\section{CONCLUSIONS}

As GPs believe that the postpartum contraceptive needs of the majority of women are being met by the 6-week checkup, it is arguable that future interventions should focus on younger women, and women from more deprived areas who may need more support in order to access services, and be less wellinformed about the various contraceptive options available to them. Future research should therefore attempt to gather the views and experiences of health visitors about their role in the provision of postpartum contraception, now that these data have been collected for midwives and GPs in the Lothian region.

Acknowledgements The authors gratefully acknowledge the support of Alison Craig (nurse consultant) in assisting with contacting GPs, and Kirsty McCance for assistance with qualitative research. The authors want to thank all the GPs who agreed to be interviewed and gave up their time willingly.

Competing interests Sharon Cameron has received research funding from HRA Pharma and Pfizer, and has been European Scientific advisory board member to Exelgyn.

Ethics approval NHS Lothian Research and Development.

Provenance and peer review Not commissioned; externally peer reviewed.

\section{REFERENCES}

1 Births in England and Wales 2013. http://www.ons.gov.uk [accessed 17 July 2014].

2 McDonald EA, Brown SJ. Does method of birth make a difference to when women resume sex after childbirth? BJOG 2013;120:823-830.

3 Jackson E, Glasier SJ. Return of ovulation and menses in postpartum nonlactating women: a systematic review. Obstet Gynecol 2011;117:657-662.

4 Information Services Division (ISD) Scotland. Breastfeeding Statistics. Financial Year 2011/12. 2012. https:/isdscotland. scot.nhs.uk/Health-Topics/Child-Health/Publications/2012-1030/2012-10-30-Breastfeeding-Summary.pdf?48888796568 [accessed 14 July 2014].

5 McCance K, Cameron S. Midwives' experiences and views of giving contraceptive advice and providing long-acting reversible contraception: a qualitative study. J Fam Plann Reprod Health Care 2014;40:177-183.

6 British Pregnancy Advisory Service. Mumsnet/bpas survey shows gaps in contraception care for new mums - unclear advice to breastfeeding women raises risk of unplanned pregnancy. 2012. http://www.bpas.org/bpasknowledge.php? year $=2012 \&$ npage $=0 \&$ page $=81 \&$ news $=543$ [accessed 19 April 2014].

7 Department of Health. Abortion Statistics, England and Wales: 2013. 2014. https://www.gov.uk/government/uploads/system/ uploads/attachment_data/file/319460/Abortion_Statistics_ England_and_Wales_2013.pdf [accessed 14 July 2014].

8 Wellings $\mathrm{K}$, Jones $\mathrm{KG}$, Mercer $\mathrm{CH}$, et al. The prevalence of unplanned pregnancy and associated factors in Britain: findings from the third National Survey of Sexual Attitudes and Lifestyles. Lancet 2013;382:1807-1816.

9 Lakha F, Glasier A. Unintended pregnancy and use of emergency contraception among a large cohort of women attending for antenatal care or abortion in Scotland. Lancet 2006;368:1782-1787.

10 Barber JS, Axinn WJ, Thornton A. Unwanted childbearing, health and mother-child relationships.J Health Soc Behav 1999;40:321-257.

11 Smith GCS, Pell JP, Dobbie R. Interpregnancy interval and risk of preterm birth and neonatal death: retrospective cohort study. BMJ 2003;327:313-318.

12 McLoone P. Carstairs Scores for Scottish Postcode Sectors from the 2001 Census. Glasgow, UK: University of Glasgow, 2004.

13 Braun V, Clarke V. Using thematic analysis in psychology. Qual Res Psychol 2006;3:77-101. 
14 Holloway I, Wheeler S. Qualitative Research in Nursing and Healthcare (3rd edn). Oxford, UK: Wiley-Blackwell, 2013.

15 National Institute for Health and Care Excellence (NICE). Routine Postnatal Care of Women and Their Babies. NICE Clinical Guideline 37. 2006. https://www.nice.org.uk/guidance/ cg37 [accessed 15 July 2014].

16 Glasier A, Logan J, McGlew T. Who gives advice about postpartum contraception? Contraception 1996;53: 217-220.

17 Scottish Government. Sexual Health and BBV Framework 2011-2015. 2011. http://www.scotland.gov.uk/Resource/Doc/ 356286/0120395.pdf [accessed 15 July 2014].

18 Department of Health. A Framework for Sexual Health Improvement in England. 2013. http://www.gov.uk/ government/uploads/system/uploads/attachment_data/file/ 142592/9287-2900714-TSO-SexualHealthPolicyNW_ ACCESSIBLE.pdf [accessed 15 July 2014].

19 National Institute for Health and Clinical Excellence. Contraceptive Services with a Focus on Young People up to the Age of 25. NICE Guideline PH51. 2014. http://www.nice.org. uk/guidance/PH51 [accessed 15 July 2014].

20 Lader D. Opinions Survey Report No. 41. Contraception and Sexual Health, 2008/09. London, UK: Office for National Statistics, 2009.
21 Trussell J. Contraceptive efficacy. In: Hatcher RA, Trussell J, Stewart F, et al. (eds), Contraceptive Technology (20th revised $e d n)$. New York, NY: Ardent Media, 2007:22-34.

22 Faculty of Sexual \& Reproductive Healthcare. UK Medical Eligibility Criteria for Contraceptive Use (UKMEC 2009). 2009. http://www.fsrh.org/pdfs/UKMEC2009.pdf [accessed 6 June 2014].

23 Smith KB, Van Der Spuy ZM, Cheng L, et al. Is postpartum contraceptive advice given antenatally of any value? Contraception 2002;65:237-243.

24 Ogburn JA, Esprey E, Stonehocker J. Barriers to intrauterine device insertion in postpartum women. Contraception 2005;72:426-429.

25 Heller R, Cameron S, Briggs R, et al. Postpartum contraception: a missed opportunity to prevent unintended pregnancy and short inter-pregnancy intervals. J Fam Plann Reprod Health Care 2016;42:93-8.

26 Tocce KM, Sheeder JL, Teal SB. Rapid repeat pregnancy in adolescents: do immediate postpartum contraceptive implants make a difference? Am J Obstet Gynecol 2012;206:481. e1-481.e7.

27 Lewis LN, Doherty D, Hickey M, et al. Implanon as a contraceptive choice for teenage mothers: a comparison of contraceptive choices, acceptability and repeat pregnancy. Contraception 2010;81:412-426. 